\title{
THE CALIBRATION SYSTEM FOR THE DELPHI BARREL RICH DETECTOR
}

P. Adrianos, E.G. Anassontzis, P. Ioannou, G. Kalkanis,

S. Katsanevas, I. Kontaxis, C. Kourkoumelis, S. Nounos,

P. Preve, L.K. Resvanis, P. Spentzouris, G. Voulgaris

Physics Laboratory, University of Athens, GR-10680 Athens, GREECE 
THE CALIBRATION SYSTEM FOR THE DELPHI

BARREL RICH DETECTOR

P.Adrianos, E.G.Anassontzis, P.Ioannou, G.Kalkanis, S.Katsanevas, I.Kontaxis, C. Kourkoumel is, S.Nounos, L.K.Resvanis, P.Spentzouris, G.Voulgaris

Physics Laboratory, University of Athens, GR-10680 Athens, GREECE

\section{ABSTRACT}

We have designed, constructed and installed a system that makes an accurate $3-D$ map of the electron drift velocity and measures the space charge distorting effects in the TPC's of the DELPHI Barrel RICH Detector. Details of the design considerations, construction and production are given.

\section{INTRODUCTION}

The Barrel Ring Cherenkov Imaging Detector (RICH) of the DELPHI experiment (fig. 1) at the LEP collider at CERN is designed to identify protons, pions and kaons for momenta between 3 and $25 \mathrm{GeV} / \mathrm{c}$ [1], following the pioneering work of Ypsilantis and Seguinot [2]. It will cover a region of $0 \circ<\varphi<360^{\circ}$ and $46^{\circ}<\theta<134^{\circ}$ where $\varphi$ is the azimuthal angle about the LEP beams and $\theta$ is the polar angle. The Barrel RICH detector is composed of two 
identical cylindrical pieces that are symmetric about a plane perpendicular to the beams on the beam crossing point. Particles crossing the two types of Cherenkov radiators of the Barrel $\mathrm{RICH}$ (liquid and gas) produce Cherenkov photons. These photons ionize a photosensitive gas and produce electrons. The determination of the coordinates of those electrons parallel to the beam axis follows the Time Projection Chamber principle while the other two coordinates are measured with a standard MWPC.

Forty eight drift tubes $(150 \times 33 \times 7) \mathrm{cm}^{3}$ are used as small T.P.C.'s (called "drift tubes" for the rest of this paper). The long dimension of each tube (drift axis) is along the z-axis of the Barrel RICH (which is parallel to the beams). The $x$-axis is along the width of the tube and the $y$-axis is along its depth (shortest dimension). The drift tubes are made out of synthetic fused quartz plates glued together. A multiwire proportional chamber is used as the single electron detector. The $x$ position is read out by the wires (with a spacing of $2.62 \mathrm{~mm}$ ) and the $y$ by cathode pads of $4.5 \mathrm{~mm}$ width. The third coordinate, the time to drift from the H.V. side (designed for high voltages up to $200 \mathrm{kV}$ ) to the MWPC is read out by a time digitizer (LTD) [3] with a least bit count of 8nsec. The gas mixture is Methane/Ethane $(75 \%-25 \%)$ with TMAE (Tetrakis-diMetyl-Amino-Ethene) [4] as the photoionizing agent.

Whenever large drift distances are used, one has to 
worry about space charge distortions [5] caused by positive ions that are formed by amplification at the MWPC. Those distortions may affect the electron drift velocity and the detected coordinates of the electron. In a large gas volume containing a photoionizing agent like TMAE, the problem is more important, especially in an $e^{+} e^{-}$ storage ring like LEP, because of the possibility of the detector getting sprayed by large amounts of synchrotron radiation, coincident in time with the interesting event. It is therefore very important to have a continuously updated and accurate map of the electric field.

The work of T.Ypsilantis and J.Seguinot [6] has shown, that if the transfer field to the detector is gated, the space charge effects are important only near the walls of the drift vessel, because of the surface charges that built up there. In the first few millimeters from the wall the distortions in the $x$ coordinate can be as large as $5 \mathrm{~mm}$. They further showed that distortions also exist in the other two directions ( $y$ and $z$ ). Preliminary studies that were performed by the University of Athens group [7], using the CX prototype [8], confirmed the above. The gas radiator rings are produced mainly in the mirror side of the drift tube where they are sensitive mainly to $x, z$ error. The liquid radiator rings are produced mainly on the opposite side of the drift tube (near the 1 iquid radiator) and are very sensitive to the $y$ 
error as well, due to the large parallax caused by the large angle that the photon directions make with the normal.

It was decided to produce a calibration system that would use photons to ionize the TMAE inside the drift tubes. The position of these "fiducial" photon sources, with respect to the drift tubes, should be known accurately and should be compared regularly to the position of the online detected electrons, generated by the flashing of the calibration system.

\section{REQUIREMENTS}

The calibration system to be designed should:

a) give an accurate 3-dimensional map of the electron drift velocity

b) give as much detailed information as possible in order to unfold and correct for the 3-dimensional space charge distorting effects, which are worst near the walls of the drift tube

c) have the intrinsic dynamic rate capability to monitor, during the course of a run, changes in the distribution of space charges which are caused by changes in the running conditions of the stored beams.

All the above should be achieved without introducing any "dead" space in the detector, without disturbing the uniformity of the $200 \mathrm{kV}$ design electric drift field and without introducing an appreciable error contribution to the overall accuracy of the final Cherenkov ring 


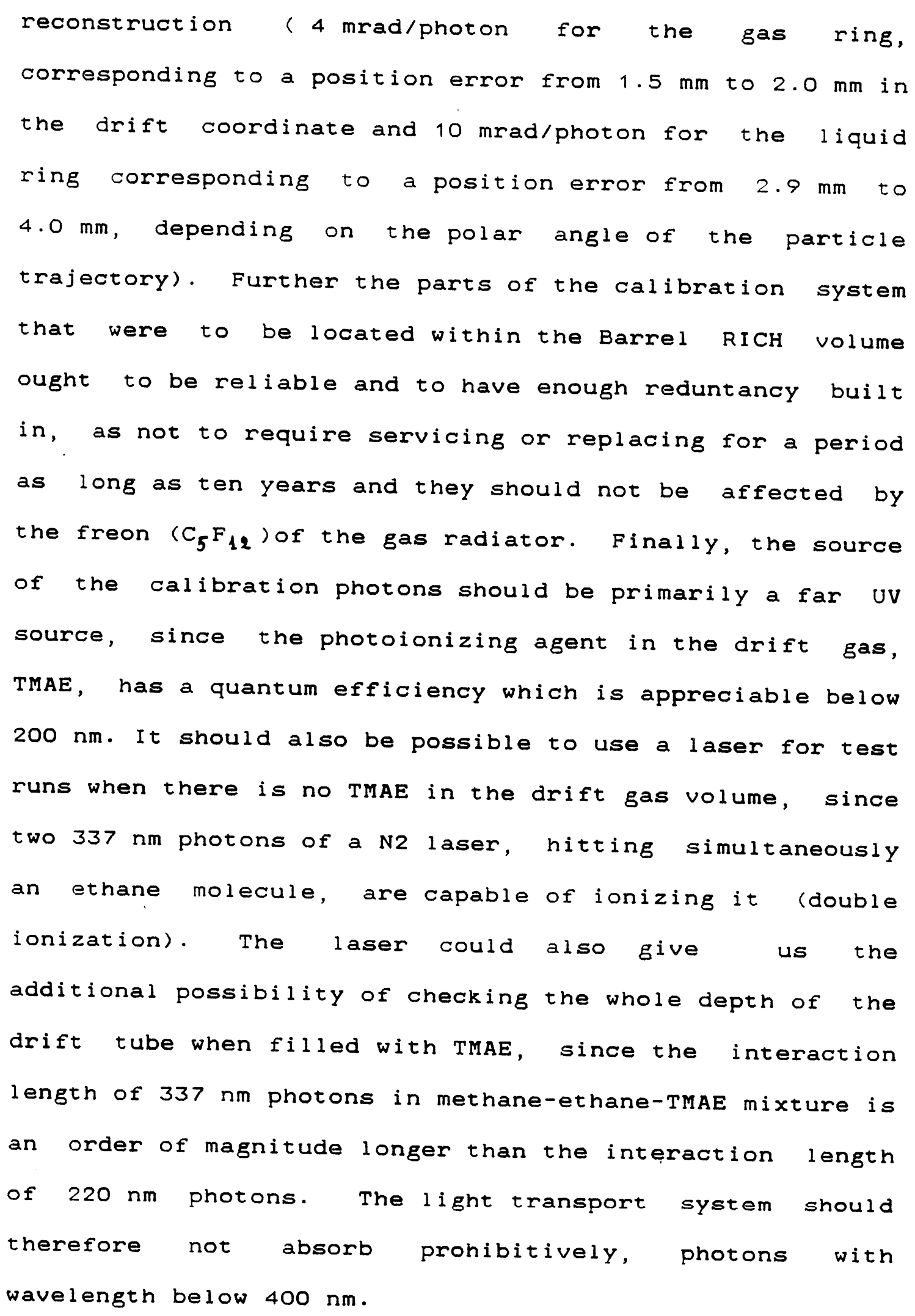




\section{DESIGN CONSIDERATIONS}

In order to fulfill the above requirements it was
decided to place the photon source outside the DELPHI
detector where, it would be easily accessible for servicing and possible modifications. There, the DELPHI stray magnetic field is a few tens of Gauss and the discharge of the sparking UV-light source would not cause any RF pickup noise to the rest of the detector. The distance from the source to the Barrel RICH "end flange" is at most fifteen meters (allowing for gentle bends around the numerous obstructions) and then a maximum of another two meters distance to reach the various points inside the Barrel RICH. The end flange, separates the gas volume of the Barrel RICH from the exterior, so any connection through it must be gas tight. The calibration light source and its light distribution unit is the subject of a different study [9]. Here, the calibration system inside the Barrel RICH volume will be described in detail and for the remainder of this paper the term "source" will be used to mean the light reaching the Barrel RICH end flange.

The mechanical unit of the Barrel RICH detector is a "bitube" (fig. 2), i.e. a set of two drift tubes and two "wireframes" held together by a structure made of Stresalit, so each half of the Barrel RICH is composed of 12 bitubes. The aim of the wireframes is to shape the electric field, in order to achieve the required electric 
field homogeneity inside the drift tubes. They are located $12 \mathrm{~mm}$ above each drift tube and parallel to it. Each wireframe is made out of Rohacel and Kevlar, with two beams $150 \mathrm{~cm}$ long and $3 \mathrm{~cm}$ wide and six stiffening ribs $29 \mathrm{~cm}$ long and $3 \mathrm{~cm}$ wide that occupy optically inactive parts of the detector. The thickness of the frame is $15 \mathrm{~mm}$ and each side of the wireframe is equiped with $100 \mu \mathrm{m}$ wires stretched parallel to the ribs every $6 \mathrm{~mm}[1]$. Each bitube with the corresponding pair of wireframes can only be inserted or removed as a unit from the Barrel RICH through an appropriate opening in the end flange. This is why it was decided that one source should supply light for both the drift tubes of each bitube unit. The easiest way to transport light from the source to the drift tubes is to use a gas tight optical coupler and to distribute the light to the various calibration points using UV light transmitting fibers. The fibers are streamed in the space between the wireframes and then they are fanned out over the ribs of each wireframe where their ends are placed, together with the "terminal optical system" (see next section), inside holes drilled into the ribs. The use of the ribs as the support structure of the calibration system does not introduce any dead space in the detector. Five ribs per frame are used and nine holes are drilled on each rib with each hole occupied by a terminal optical system and its fiber. The holes of each 
rib are symmetric with respect to the middle point of the rib, densely populating the edges, where the maximum distortions are expected (fig. 3a). They are staggered in the $z$ direction in order to facilitate the resolution of the light spots. The $x, z$ location of the center of intensity of each light spot gives the center of the $x, z$ coordinates of the calibration signals. Ninety (90) fibers were used for each bitube ( $f i g$. 3b), held together in a bunch at the light receiving end. Extensive test in the ATHENS set up [10], which simulates the Barrel RICH electrostatic configuration, showed that no discharge were caused by the presence of the fibers, up to the design electric field of $200 \mathrm{kV}$.

CONSTRUCT ION

\section{i) The fibers}

The design of the end flange is such that the only space available to feed through the fibers, with a gas tight feed through, is a hole $14 \mathrm{~mm}$ in diameter for every pair of drift tubes. Fiber availability and the size of the hole constrain the diameter of the fiber core to $200 \mu \mathrm{m}$ or $600 \mu \mathrm{m}$. Two companies were located that make UV transmitting fibers, "Quartz et Silice", in France and "Showa", in Japan. The fibers produced by Quartz et Silice are clad with silicon, while the showa fibers are clad with quartz. The transmission of the fibers in the far UV was measured using a monochromator. The results of the measurements are shown in fig.4. Clearly all three fibers 
were adequate for our needs. So space considerations became decisive. The minimum radius of curvature for the $600 \mu \mathrm{m}$ fiber without putting undue mechanical strain on the fiber is $100 \mathrm{~mm}$, while for the $200 \mathrm{\mu m}$ is $10 \mathrm{~mm}$. This minimum radius of curvature of the $600 \mathrm{\mu m}$ fiber, excludes its use on the wireframes, since the opening in the end flange, through which the drift tube assembly is to be introduced inside the Barrel RICH, is only a couple of centimeters bigger than the drift tube assembly. Further, after various polishing tests on the light receiving end of the fiber bunch, it was decided to use a fiber with quartz cladding, because the quartz cladding made polishing more uniform as compared to fibers with silicon cladding. Thus, by elimination, it was decided to use the $200 \mathrm{\mu m}$ Showa quartz clad fiber. For each bitube a bunch of 91 fibers (45 for each one of the two drift tubes plus one spare) was produced and the light receiving end of the bunch, after polishing, was placed inside a home made gas tight feed through (fig. 5) and illuminated by a convex fused silica lens of $6 \mathrm{~mm}$ diameter and $10 \mathrm{~mm}$ focal length. The illumination coverage that the bunch received when a $600 \mu \mathrm{m}$ fiber supplied the light on the other side was measured using a monochromator. Two wavelengths were used, $220 \mathrm{~nm}$ i.e. typical wavelength for single ionization of the TMAE and $337 \mathrm{~nm}$ i.e. N2 laser wavelength for doubleionization. Typical curves are shown in fig. 6 . Clearly 
the bunch is illuminated fairly uniformily.

ii) The terminal optical system

The light emmiting end of each fiber was glued inside a plastic pipe holder, made of "Derlin". The other side of the holder supports a plano-convex lens made out of fused silica with a diameter of $3.5 \mathrm{~mm}$ and a focal length of $8 \mathrm{~mm}$ (details are shown in fig. 7 ). The distance of each lens to the corresponding light emmiting end was tuned to produce a parallel light beam in the violet wavelength region and was secured in place with instant glue (Powabond). An epoxy glue (Araldite) was then used to produce a permanent bond. Typical light beam spreads, from the above described terminal optical system, at a distance of $26 \mathrm{~mm}$ from the lens are shown in fig. $8 a$ for $\lambda=200 \mathrm{~nm}$ and $\lambda=337 \mathrm{~nm}$, while the spread for a distance of $56 \mathrm{~mm}$ is shown in fig. 8b. These distances correspond to a depth, inside the drift tube, of 1 and 3.5 interaction lengths of TMAE of $35^{\circ} \mathrm{C}$, respectively. In these measurements care was taken so that the light receiving end of the bunch was illuminated by a $600 \mathrm{\mu m}$ fiber as in the final system. The light intensity from each fiber (equipped with the terminal optical system) was measured in a monochromator for $\lambda=220 \mathrm{~nm}$ and $\lambda=337 \mathrm{~nm}$. Fig. 9 is a histogram of the measured light output intensity from all the fibers of a typical bunch. Each fiber bunch was mounted on a bi-frame and the terminal optical systems were inserted in the 45 holes of each frame and were secured with Araldite. 
Care was exercised in choosing the fibers that were placed in adjacent holes to be of comparable light intensity output (no more than 50\% difference), so that, they would not flood each other.

iii) Measurement of the light spot; errors

It was decided to produce one calibration map for the gas radiator Cherenkov rings (mirror side of the drift tube) and another one for the liquid radiator Cherenkov rings (liquid radiator side of the drift tube). Clearly the calibration map for the middle of the tube can be reconstructed by interpolation. The position and direction of each light beam emerging from each fiber, after being placed in its final position in the wireframe, had to be known with such a precision as not to influence significantly the overall error in the measurement of the Cherenkov rings. A measuring table was specially constructed, using an $x-y$ mechanicalloptical digitizer (CEFAL with "MCB Coder-optique" digitizers) that was used in the past on a Bubble Chamber film image plane digitizer. The least count accuracy is $30 \mu \mathrm{m}$. A square photoconductive diode (PINC1ODG), with four output leads in the middle of each of the sides of the square, was used to measure the center of the intensity of the light emitted by the fiber-lens system. The diode was finely displaced around each hole until the simultaneous zero crossing of the voltage difference between the opposite 
lying leads was achieved. The digitization system was then triggered and the position of the light spot center of intensity was recorded through CAMAC, by a SUPER-CAVIAR microcomputer. First the position of all light spots was recorded on a plane $30 \mathrm{~mm}$ from the bottom of the wireframe. Then the same procedure was repeated at a distance of $60 \mathrm{~mm}$ from the bottom of the wire frame, i.e. corresponding to roughly one TMAE (of temperature $35^{\circ} \mathrm{C}$ ) interaction length from the top and the bottom of the drift tube respectively.

The error on the spot position is due to:

a) An error of $\pm 80 \mu \mathrm{m}$ in the determination of the measuring table constants

b) An error of $\pm 30 \mathrm{\mu m}$ due to the least count of the digitizing system

These two errors combined in quadrature give an overall spot error of $\pm 85 \mu \mathrm{m}$. This was independently confirmed by constructing a model bar with two holes at each end, made on a digital milling machine. The holes were instrumented with light spots and the relative position of the light intensity centers was measured, all over the measuring table and for many orientations of this model bar.

For an absolute position measurements one must take further in account:

c) An error of $\pm 140 \mu \mathrm{m}$ in the location of the reference 


\begin{abstract}
points. (The reference points were attached on the measuring table and their position relative to the positioning pins was measured on a digital milling machine. The positioning pins are a pair of pins, $4 \mathrm{~mm}$ of diameter, on each wireframe with corresponding
\end{abstract}

holes in the bitube structure)

d) The tolerances of $\pm 50 \mathrm{~km}$ in the construction of the wireframe, i.e. in the distance between the edges of the frame and the positioning pin

Thus the total error in the absolute position is less than $\pm 200 \mathrm{\mu m}$

This error is insignificant compared to the positioning error of the wireframe with respect the MwPC, the MWPC $x$ and $t$ ime resolution, the diffusion and parallax of the electrons inside the drift tube [6].

\title{
CONCLUSIONS
}

We have designed and produced (fig.10) 24 calibration systems in the far UV for the 24 Barrel RICH bitubes. Each system calibrates two drift tubes, using light transmitted by 45 fibers for each drift tube. Each calibrating light spot produces fiducial points inside the drift tubes. The $x, z$ and $y$ positions of the fiducial points were recorded on a measuring table and thus known with an absolute precision of better than $\pm 200 \mathrm{\mu m}$, which introduces an error less than $7 \%$ of the overall reconstruction accuracy. This mapping procedure was repeated for each of 
the calibration systems installed in the DELPHI Barrel RICH detector. The maps will be used to compare the position of the fiducial points with the on-line detected position of the light spots, deduced from the drift electrons. This way, the space distorting effects will be determined and corrected and the performance of the Barrel RICH detectors will be monitored continuously.

\section{ACKNOWLEDGMENTS}

We would like to thank T.Ypsilantis and J.Seguinot for many stimulating discussions and valuable advice. We would also like to thank our colleagues of the Barrel RICH Construction Group for many very useful discussions; special thanks go to M.Davenport, W.Klempt, E.Rosso and B.Goret. We are also indebted to A.Apostolakis for loaning us the $x-y$ digitization system. 
R E $\quad F \quad E \quad R \quad E \quad N \quad C \quad E S$

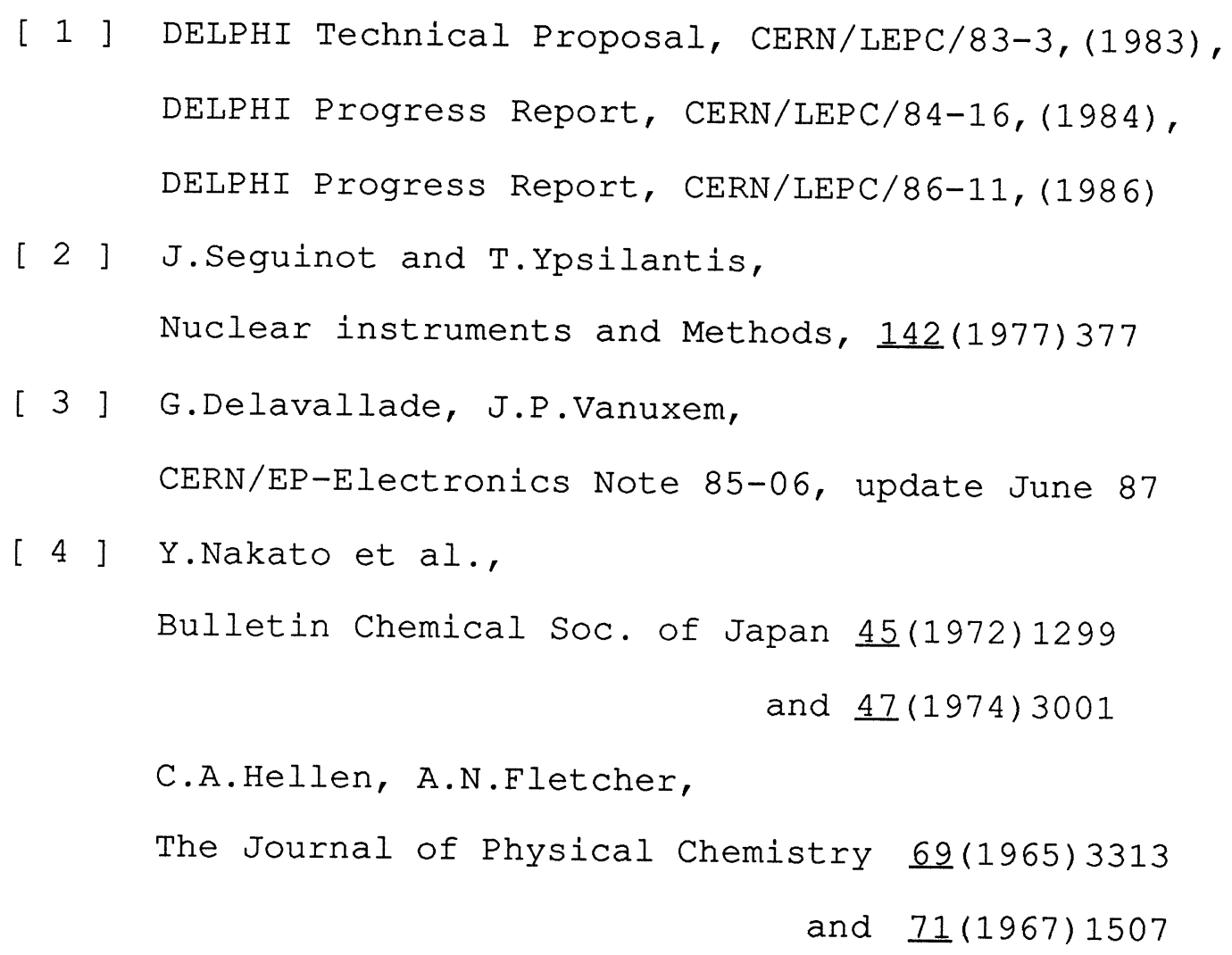


Fig.1 The Barell RICH DELPHI detector. Partial cross section along the beams of LEP.

Fig.2 Schematic of the DELPHI Barrel RICH detector with a bitube and the calibration system

$\mathrm{Fig} \cdot 3$ a) Detail of a wireframe rib indicating the fiber positions

b) A wireframe pair with the 90 fiber positions

Fig.4 Attenuation length for the three candidate fiber types

Fig.5 The coupling of the exterior "source" fiber to the interior bunch through the gas tight feedthrough

Fig.6 Typical spread of the light intensity illuminating the light receiving end of the 90 fibers bunch, at the proper distance

Fig.7 The terminal optical system placed at the end of each of the 90 fibers

Fig.8 Typical spread of the light intensity from a terminal optical system for $220 \mathrm{~nm}$ and $337 \mathrm{~nm}$ wavelengths, a) at a distance of $26 \mathrm{~mm}$ and b) at a distance of $56 \mathrm{~mm}$ from it

Fig.9 Typical distribution of the light intensity at the terminal optical system from all the fibers of a bunch for two wavelengths: a) $\lambda=220 \mathrm{~nm}$ 
and b) $\lambda=337 \mathrm{~nm}$.

Fig.10 Photograph of a bi-frame on a support, equipped with fibers and ready to be mounted on a bitube. 


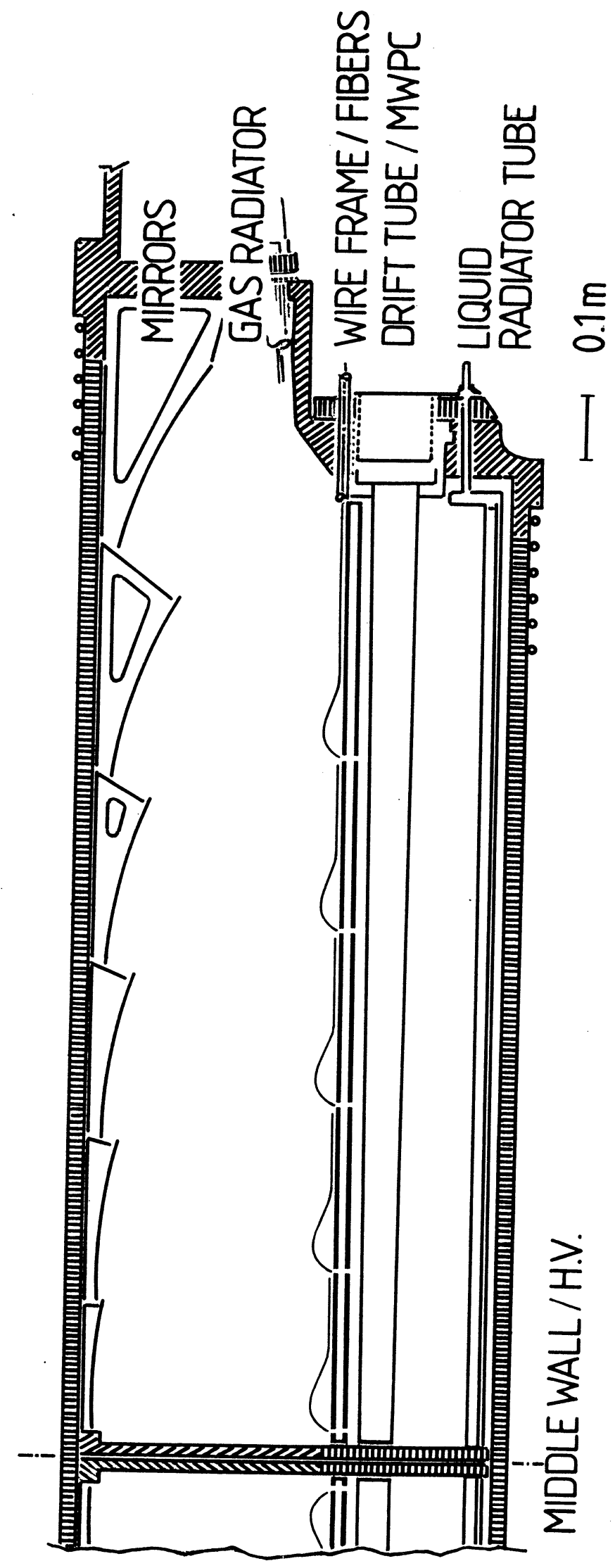




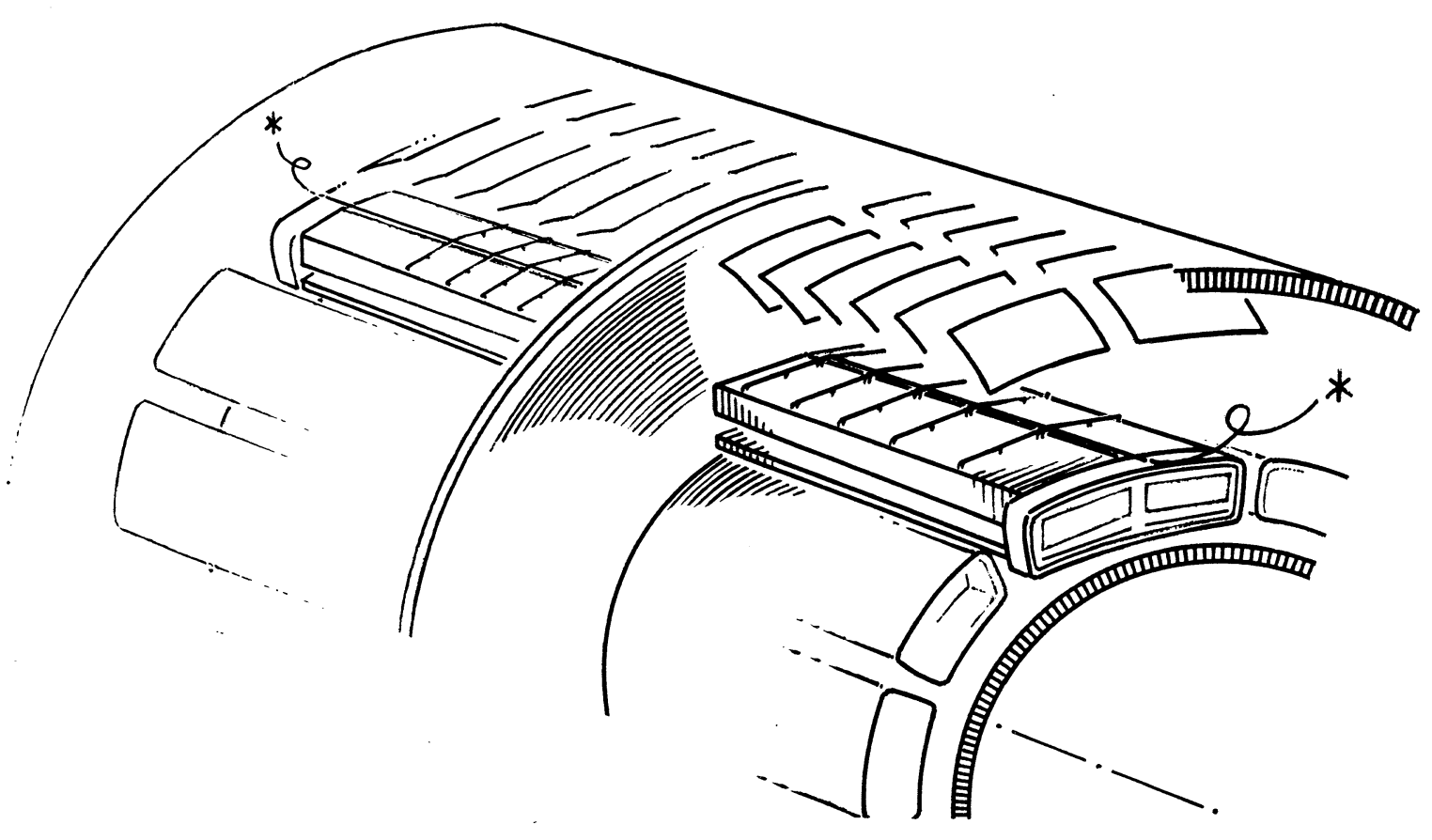

Fia. 9 


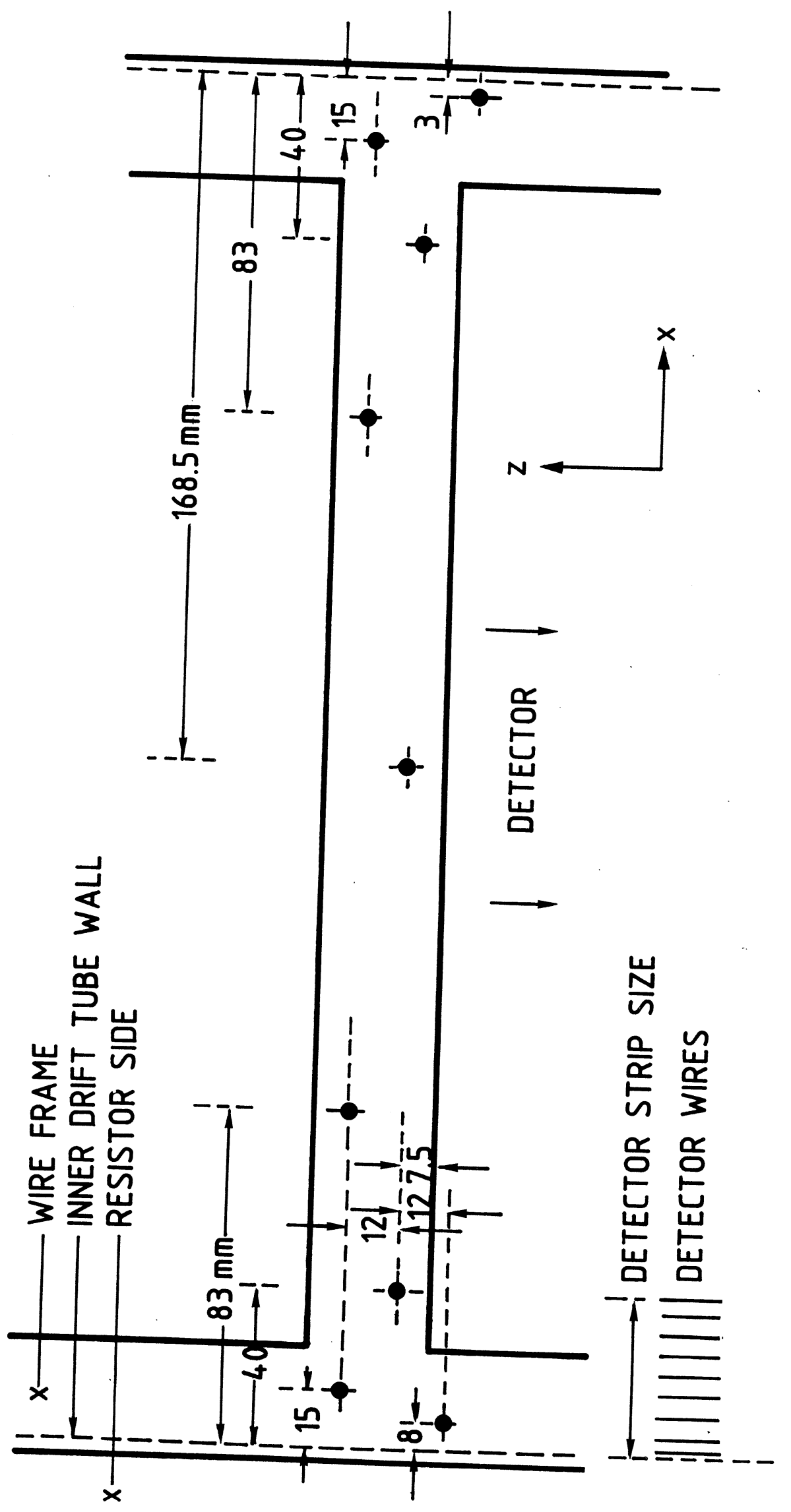




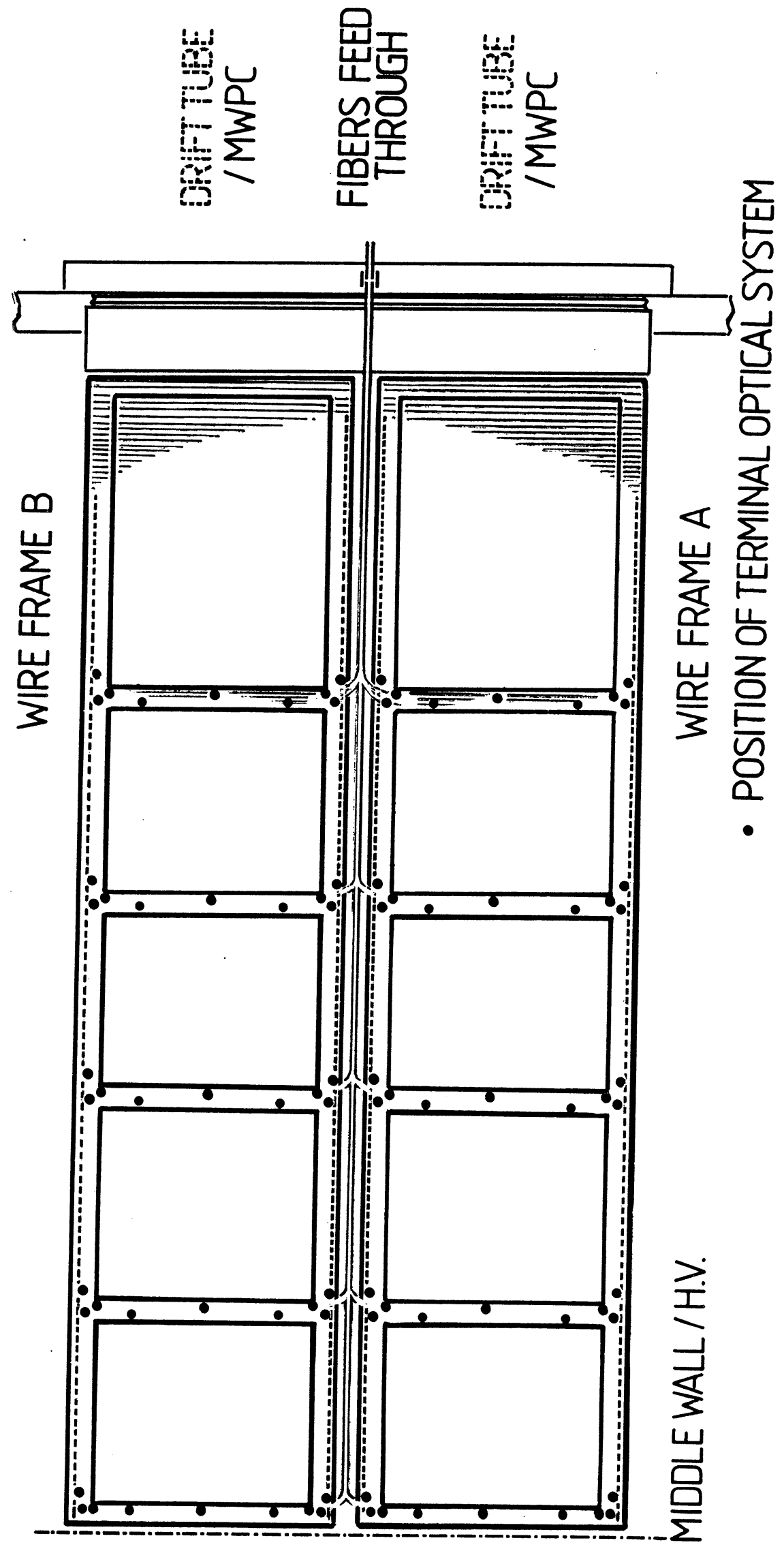




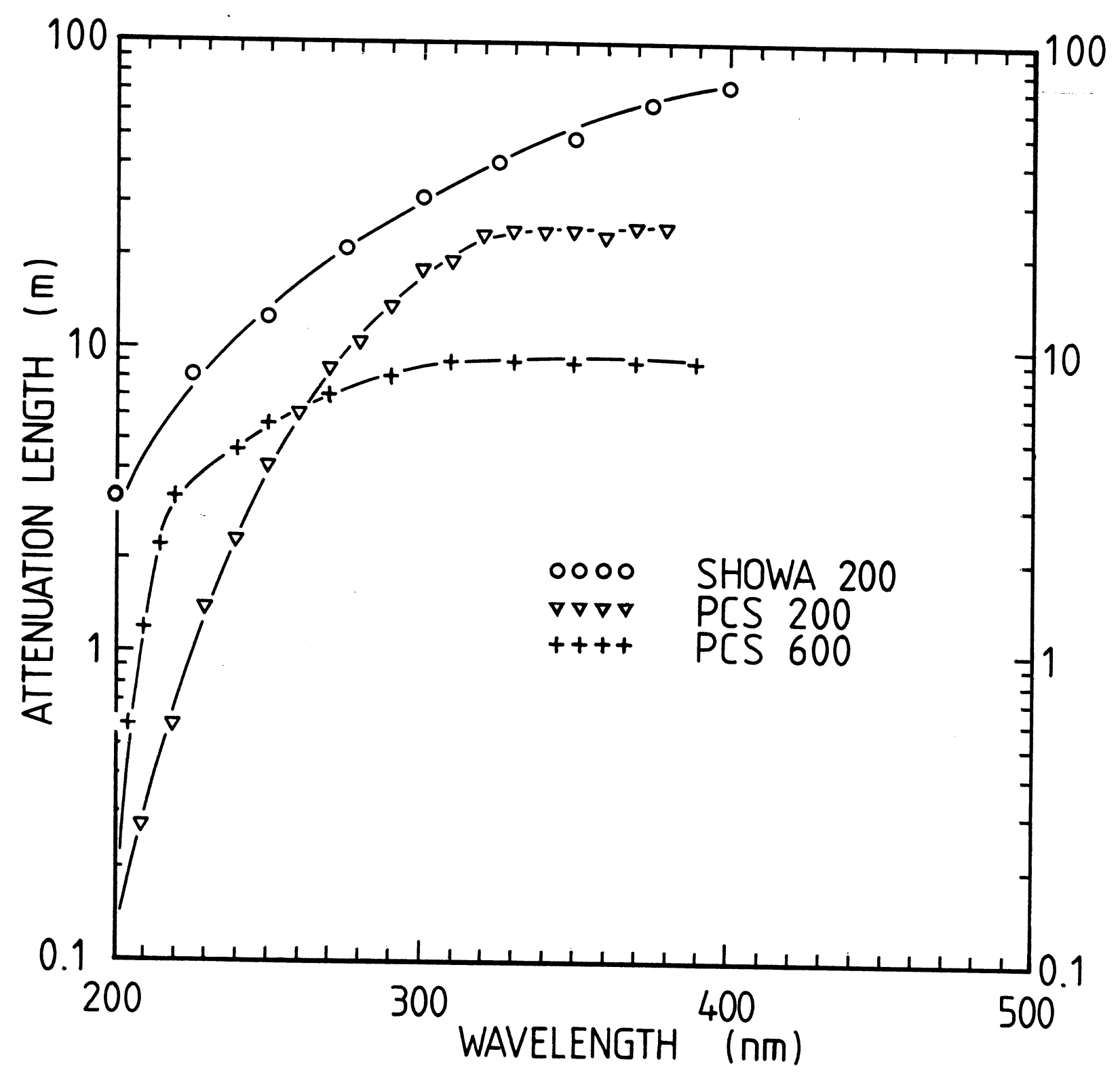

Fig. 4 


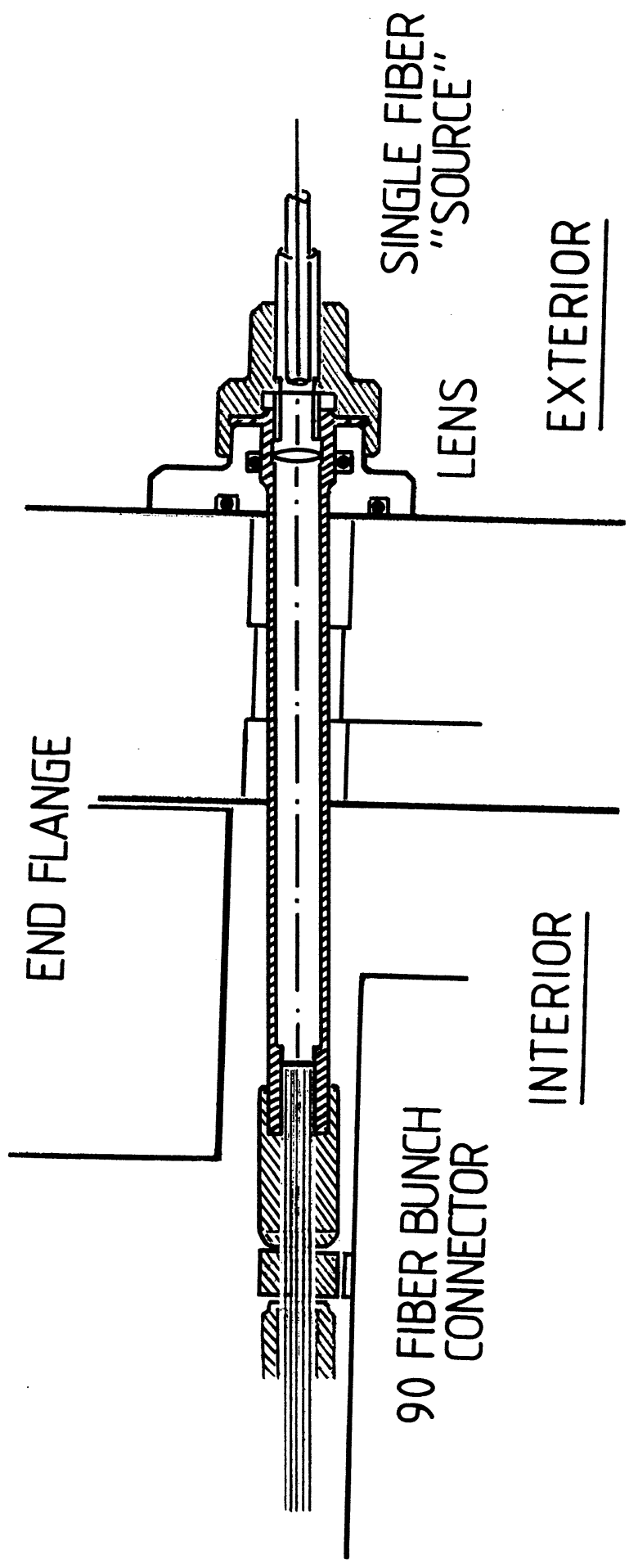




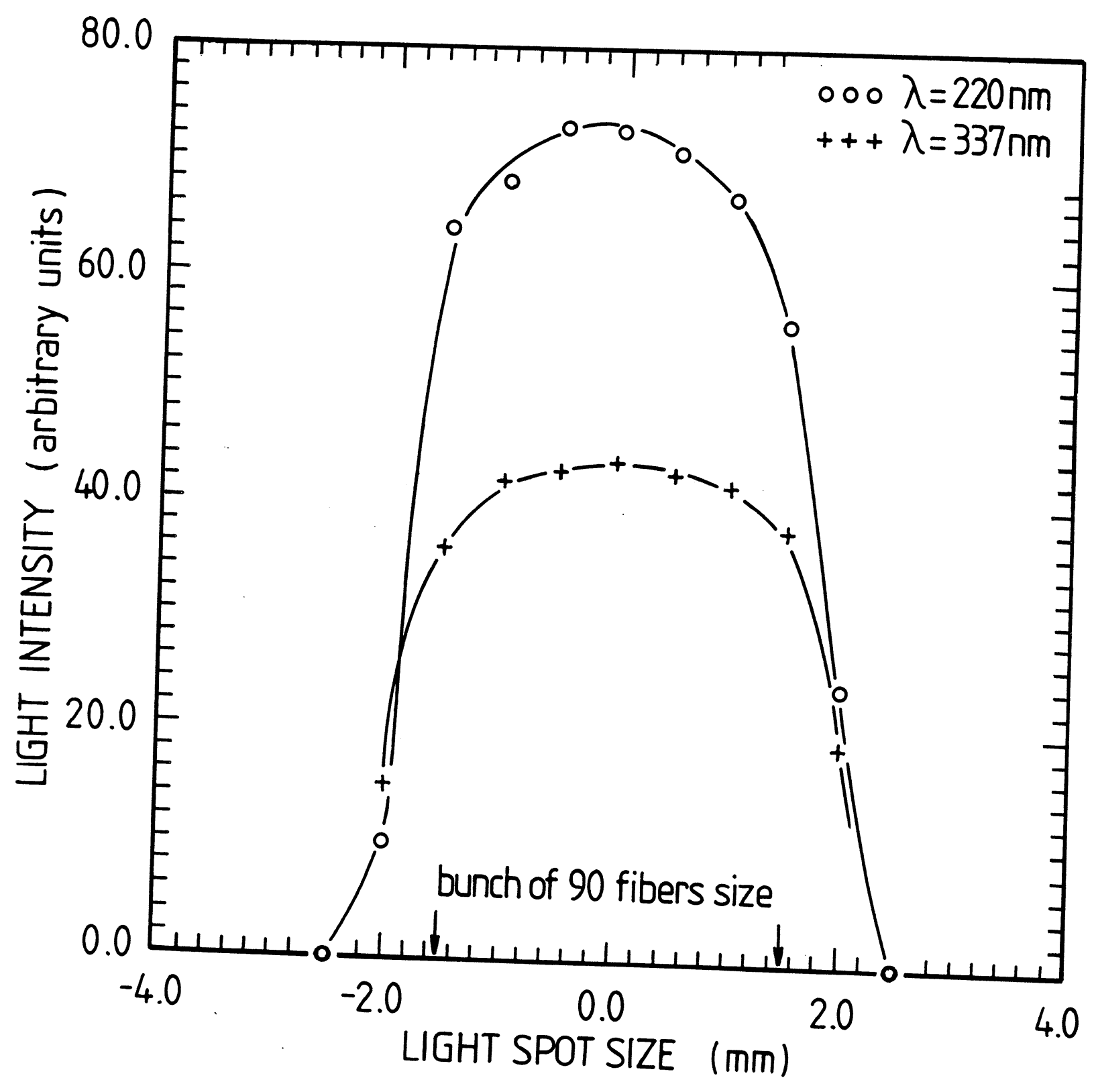

Fia. 6. 


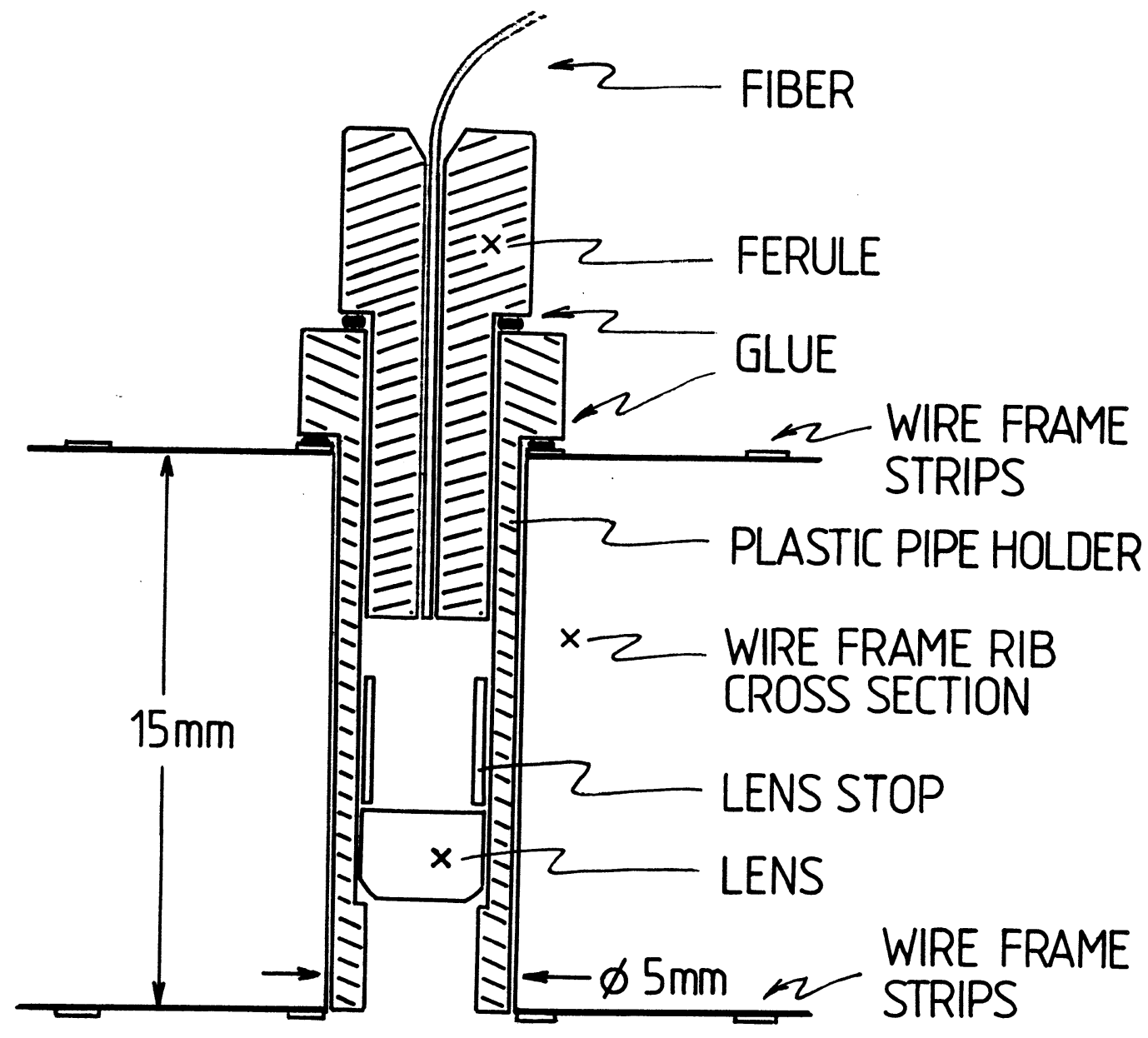

Fig. 7. 

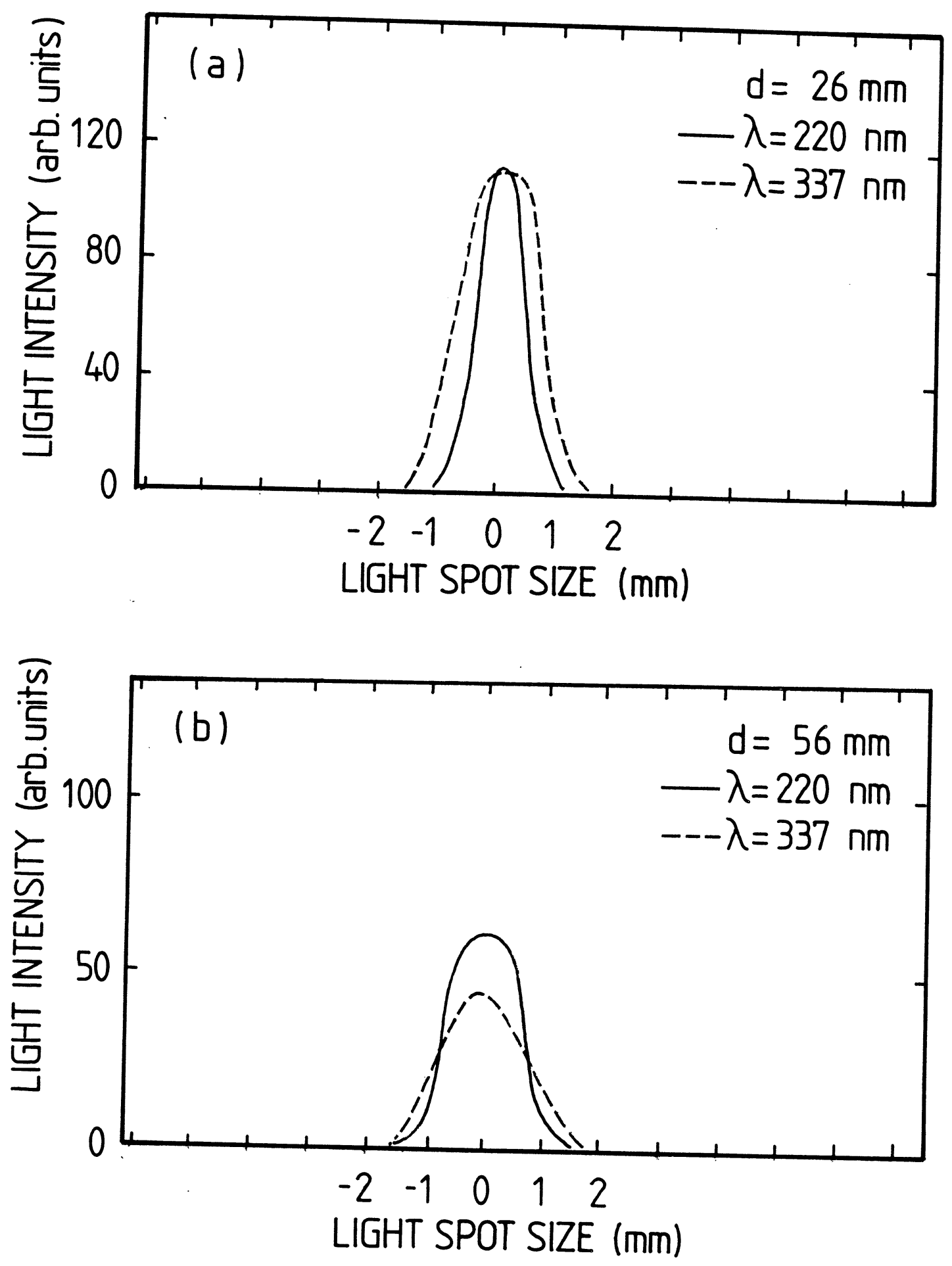

Fig. 8. 

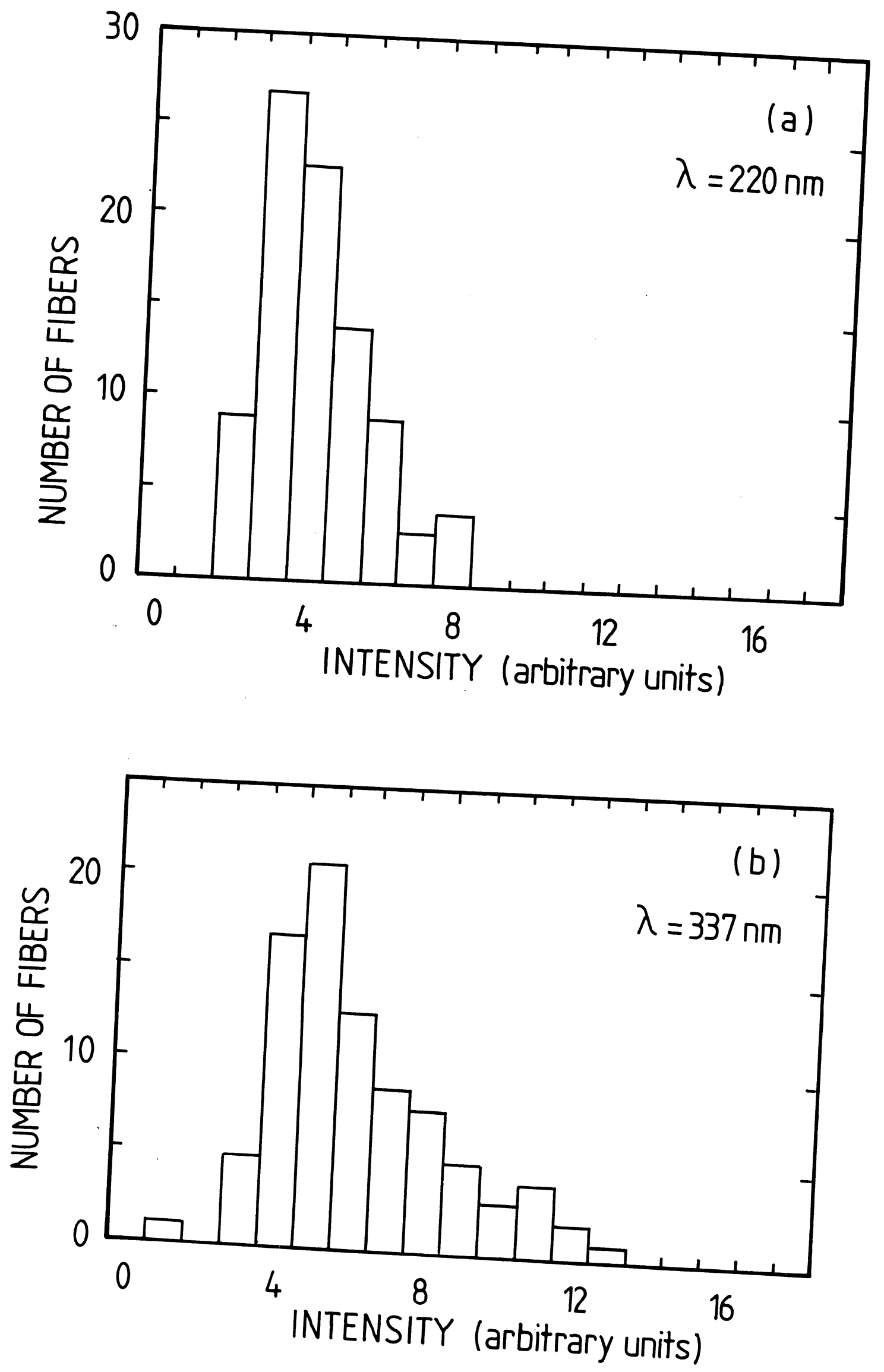


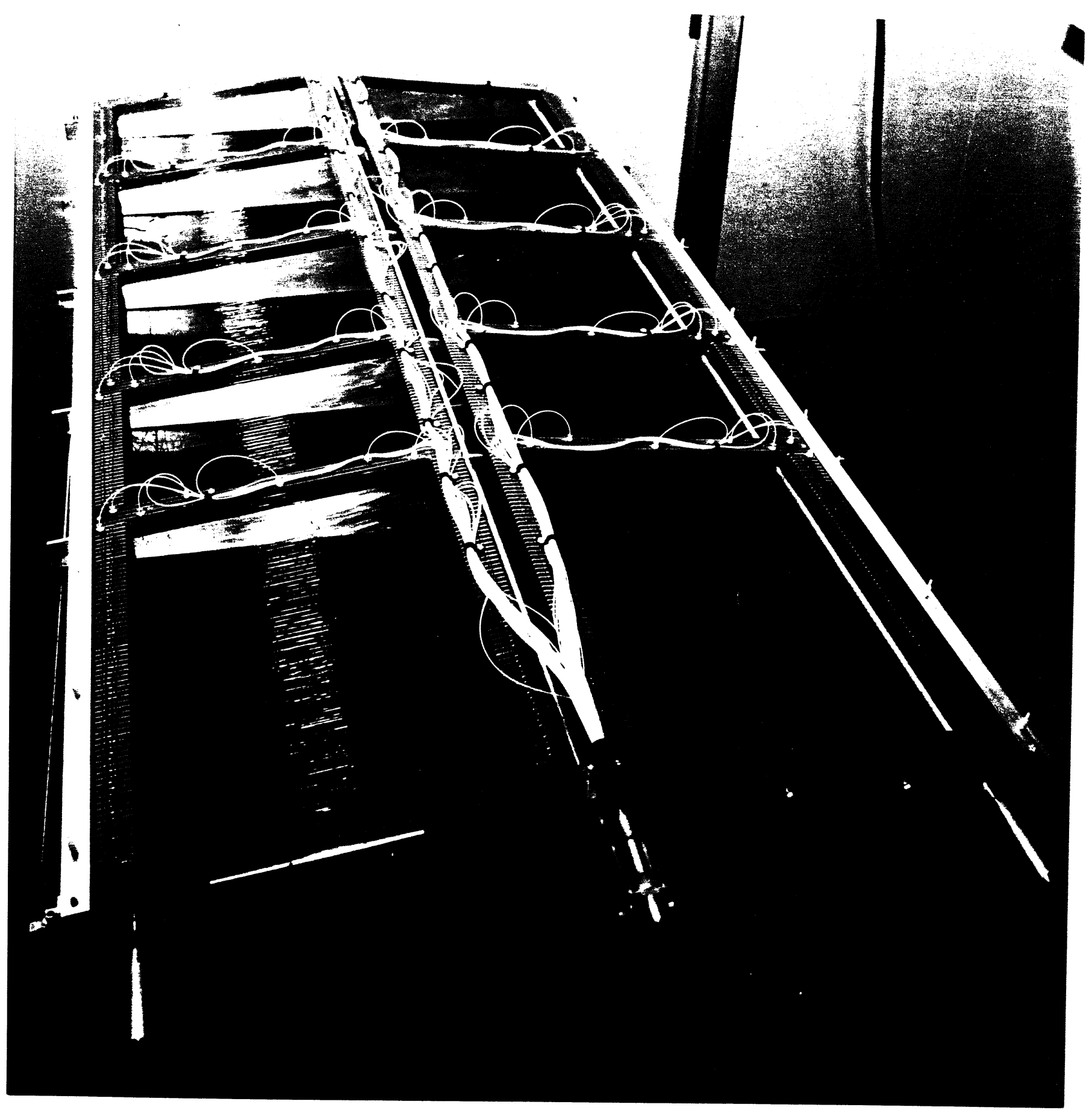

